Revue d'histoire de l'enfance « irrégulière »

Le Temps de l'histoire

$4 \mid 2002$

Images de l'enfance et de la jeunesse "irrégulières »

\title{
L'image du jugement dernier dans la salle d'audience du tribunal pour enfants de Paris
}

Une tapisserie de Georges Devêche (1946)

Jacques Bourquin

\section{OpenEdition}

\section{Journals}

Édition électronique

URL : http://journals.openedition.org/rhei/57

DOI : $10.4000 /$ rhei. 57

ISBN : 978-2-7535-1642-7

ISSN : 1777-540X

Éditeur

Presses universitaires de Rennes

Édition imprimée

Date de publication : 15 novembre 2002

Pagination : 145-155

ISSN : 1287-2431

\section{Référence électronique}

Jacques Bourquin, «L'image du jugement dernier dans la salle d'audience du tribunal pour enfants de Paris », Revue d'histoire de l'enfance «irrégulière » [En ligne], 4 | 2002, mis en ligne le 20 juin 2007 , consulté le 04 décembre 2020. URL : http://journals.openedition.org/rhei/57 ; DOI : https://doi.org/ 10.4000/rhei.57 


\section{L'image du jugement dernier} dans la salle d'audience du tribunal pour enfants de Paris : une tapisserie de Georges Devêche (1946)

Cette tapisserie qui orne toujours la salle d'audience du tribunal pour enfants de Paris fut, selon Guillaume Jeanneau, directeur de la manufacture des Gobelins depuis 1937, l'objet, en 1943, d'une commande de la ville de Paris, "préoccupée, écrit-il, de décorer la salle d'audience du Tribunal créé pour les jeunes délinquants. »(2)

C’est un jeune magistrat, Jean Chazal, contacté par Guillaume Jeanneau, qui rencontre à plusieurs reprises Georges Devêche pour le sensibiliser aux problèmes de la justice des mineurs. En 1943, Jean Chazal est responsable au ministère de la Santé de la coordination Justice-Santé-Éducation nationale qui traite des problèmes de l'enfance irrégulière. Par la suite, il fut, en 1945, juge des enfants au tribunal de la Seine. Jean Chazal sera, après la guerre, un des théoriciens au niveau national puis international de la justice des mineurs, à laquelle il a consacré plusieurs ouvrages.

Dans son article de 1947, Guillaume Jeanneau évoque un concours restreint pour l'attribution de la commande à Georges Devêche ; il n'en a pas été retrouvé trace. Selon André Devêche, frère de l'artiste, que nous avons rencontré, ${ }^{(3)}$ il n'y aurait pas eu de concours et Guillaume Jeanneau aurait proposé le nom de Georges Devêche, déjà bien connu par la manufacture des Gobelins.

À ce jour, on ne sait pas si la tapisserie a été réalisée aux Gobelins ou à Aubusson, les archives manquent, mais il semblerait que, pendant la guerre, l'activité des Gobelins ait été très réduite au profit d’Aubusson.
Jacques Bourquin $^{(1)}$
(1) Président de

l'Association pour l'histoire de l'éducation surveillée et de la protection judiciaire des mineurs (AHES-PJM).

(2) Guillaume

Jeanneau, Hyphe, janvierfévrier 1947, p. 29-30.

Il est à remarquer que le tribunal pour enfants de la Seine existait depuis 1914, en application d'une loi sur l'enfance délinquante de 1912.

(3) Entretien de Jacques Bourquin avec André Devêche, le 18/10/1999, à Neuilly. 


\section{Qui est Georges Devêche?}

Né en 1903, décédé en 1974, Georges Devêche est l'héritier d'une dynastie de décorateurs d'intérieur parisiens qui remonte à 1870 pour se poursuivre encore actuellement. Son grand-père Alexandre, son père Pierre, son frère André ont laissé des noms dans le milieu des arts décoratifs.

Georges Devêche participe avec son père et son frère à la décoration du pavillon de l'Algérie à l'exposition coloniale de 1931, puis à celle de la salle des fêtes de la mairie du XIvème arrondissement de Paris. Georges Devêche s'oriente plus particulièrement vers la tapisserie, c'est un artiste indépendant qu'il est difficile de rattacher à une école particulière, bien qu'il soit influencé par le style Arts déco des années 1930. Ses tapisseries ne sont pas sans rappeler celles de Lurçat, de Picard le Doux, bien que d'une facture plus classique, moins audacieuse.

Le Mobilier national lui commande, pendant la guerre, des cartons de tapisseries sur les "arts du feu", le "textile", œuvres qui sont réalisées à la manufacture des Gobelins. C’est à cette époque qu'il débutera son travail pour la tapisserie du tribunal pour enfants de Paris, qu'il achèvera en 1946.

Au lendemain de la guerre, Georges Devêche poursuit son activité en réalisant plusieurs tapisseries à partir de l'œuvre de Paul Claudel, L'annonce faite à Marie, Le soulier de satin. À partir de 1960, Georges Devêche se consacrera surtout à l'art du vitrail. En relation avec l'architecte A. Froidevaux, il produit de nombreux cartons dans le cadre de la restauration de la cathédrale d'Auch, des églises Sainte-Radegonde et Saint-Hilaire à Poitiers, de l'église d'Orcival en Auvergne. Il est mort à Grosrouvre, dans les Yvelines, où plusieurs vitraux de l'église lui sont dus.

\section{Circonstances de l'œuvre}

La commande de la tapisserie date de début 1943. Les deux premiers projets remis à Jean Chazal sont datés de décembre 1943.

C'est une période où le problème de l'enfance délinquante préoccupe particulièrement le gouvernement de Vichy. Les chiffres de la délinquance juvénile sont en augmentation de plus en plus rapide depuis 1940. Dès la défaite de juin 1940, l'État français, dans une logique d'en- 
cadrement et de remoralisation de la jeunesse, on parle même d'armement moral, crée les Chantiers de jeunesse puis, pour les plus jeunes, les Compagnons de France. Il faut pallier l'absence des pères tués ou prisonniers. Pour les jeunes délinquants ou en risque de le devenir, on va parler de "rééducation", de "centre prophylactique" ; les ingrédients en sont souvent des projets fondés sur le retrait de la ville, le retour au monde rural : " la nature, au moins, elle ne ment pas ", dit le maréchal Pétain. ${ }^{(4)}$

À cela s'ajoutent le respect du chef, l'autorité, le travail, l'apprentissage, la pratique du sport... C'est sur ces bases que se créent, début 1942, les premiers petits centres d'accueil ${ }^{(5)}$ pour les jeunes délinquants, auxquels on essaiera d'éviter la détention préventive. Ces centres ouverts assez souvent par des jeunes gens issus des mouvements de jeunesse, dont le scoutisme, donneront naissance à une profession à venir : les éducateurs. Une loi du 27 juillet 1942 réforme la législation sur l'enfance délinquante, qui remontait à 1912, et introduit la notion d'éducabilité du mineur délinquant en la substituant à la vieille notion de discernement, qui remontait à 1791. Ce projet d'éducabilité du mineur délinquant remontait à la fin du XIXème siècle, mais il avait fallu les campagnes de presse contre les bagnes d'enfants, de 1934 à 1937, pour voir apparaître, à la veille de la guerre, les premières tentatives de réforme de ces institutions qui accueillaient les mineurs de justice.

Vichy s'inscrivait dans ce domaine dans la continuité d'un grand courant réformiste mené depuis la dernière partie du XIXème siècle par des juristes, des philanthropes, des médecins, des hommes politiques. Cette loi de 1942 privilégiait surtout le rôle de l'observation du mineur avant la décision du tribunal des enfants, en excluant, contrairement à la loi de 1912, les mineurs de plus de 16 ans de cette amélioration législative.

Dans la lettre au maréchal Pétain qui précédait l'acte dit « loi du 27 juillet 1942 ", le garde des Sceaux, Barthélémy, introduisait son propos en écrivant : "la volonté de faire de la jeunesse française, une jeunesse forte, saine de corps et d'esprit préparée aux tâches qui élèvent les âmes, [...] un des buts principaux de la révolution nationale ".

La jeunesse délinquante, longtemps considérée comme "inamendable", était incluse dans ce courant de réforme de la jeunesse. Moins de trois ans
(4) Appel du 25 juin 1940 .

(5) Christian Sanchez, "Les Centres d'accueil et de triage de l'Éducation surveillée 1941-1950", le Temps de l'histoire, $\mathrm{n}^{\circ} 1$, 1998, p. 121-137. 
(6) Henri et Fernand Joubrel, L'enfance dite "coupable", Paris, Blond et Gay, 1946.

(7) Michel Chauvière, Enfance inadaptée :

l'héritage de Vichy, Paris, Éditions ouvrières, 1987. plus tard, alors que le régime a changé et que la libération du territoire est en cours, l'ordonnance du 2 février 1945 sur l'enfance délinquante s'inscrit, avec des nuances il est vrai, dans la continuité du texte de 1942. Dans son exposé des motifs, le garde des Sceaux de 1945, P.-H. Teitgen, écrit : " La France n'est pas assez riche d'enfants pour qu'elle ait le droit de négliger tout ce qui peut faire des êtres sains. [...] La guerre et les bouleversements qu'elle a provoqués ont accru dans des proportions inquiétantes la délinquance juvénile. [...] La République Française entend protéger efficacement les mineurs et plus particulièrement les mineurs délinquants ».

Il y a là un fort appel à la jeunesse, nécessaire pour reconstruire le pays. Les mineurs de justice ne sont pas exclus. Victimes de la guerre, il faut les rééduquer. C'est dans ce contexte, celui de la guerre, de l'occupation puis de la libération, entre ces deux textes législatifs, que G. Devêche élabore et produit sa tapisserie pour le tribunal pour enfants. C'est pendant cette même période que le juge Chazal participe au ministère de la Santé, puis au tribunal pour enfants de la Seine, à la constitution de tout un secteur d'intervention auprès de l'enfance dite "coupable", (6) que l'on qualifiera plus généralement, à la fin de la guerre, "d'enfance inadaptée". (7) Il y a la volonté d'intégrer l'enfance coupable dans le secteur des enfants en danger moral, ceux qui sont soignables, rééducables. À cela s'ajoute une idée plus ancienne, que l'enfant coupable est aussi enfant victime. Cela est d'autant plus vrai au lendemain de la guerre.

\section{Étude de la tapisserie}

Depuis les années 1930, on peut parler d'un véritable renouveau de la tapisserie, en particulier sous l'impulsion de Jean Lurçat qui découvre, en 1938, l’Apocalypse d'Angers réalisée à la fin du XIVème siècle. On assiste à un retour à la tradition médiévale, avec l'abandon des effets de perspective, une réduction de la palette des couleurs.

Il est à remarquer que Georges Devêche terminera sa tapisserie en 1946, l'année où est inaugurée à Paris, au musée des Arts décoratifs, une grande exposition qui illustre le renouveau de la tapisserie en France. G. Devêche s'inscrivait pleinement dans ce courant. En décembre 1943, il présente à Jean Chazal deux projets. 


\section{Le premier projet}

Ce premier projet [cf. illustration p.150] est intitulé par Georges Devêche : Le travail, symbole de la vie honnête. L'œuvre se construit autour de l'image de la famille, qui occupe le centre du carton dans une sorte de grand losange où figurent le père assis à table tenant sur ses genoux le plus jeune enfant, la mère debout - une image traditionnelle dans la famille paysanne - qui sert le potage, un garçonnet portant le pain, une petite fille qui s'agrippe à la jupe de sa mère. Une image pleine de sérénité, loin de la guerre, que Devêche commente ainsi dans un texte joint au carton : "Le bonheur familial comme récompense de la vie vouée au travail. » ${ }^{(8)}$

Autour de cette scène familiale, le travail est représenté en haut et à droite de l'œuvre par un laboureur, à gauche par un paysan qui sème, en bas par la moisson et un boulanger qui pétrit le pain. Le travail, la famille, deux valeurs emblématiques de l'époque qui s'inscrivent sur un fond de ruralité. La bordure de la tapisserie, en référence à la tapisserie médiévale, allie l'image à l'écriture. À droite de l'œuvre se trouvent mentionnés quatre des sept péchés capitaux : l'avarice, l'orgueil, la paresse, la colère. L'auteur a fait un choix ; ne figurent pas la luxure, peut-être peu compatible avec l'image de l'enfance, la gourmandise, trop vénielle, l'envie. Il est à remarquer que, dans un avant-projet dont nous avons quelques détails, apparaissaient la jalousie et la méchanceté, mais ce ne sont pas des péchés capitaux. À gauche de l'œuvre, quatre vertus sont inscrites : la charité, la volonté, la douceur, le courage. Tous ces vices et vertus sont représentés par des personnages féminins vêtus de longues robes. Dans son texte d'accompagnement, Georges Devêche évoque "le cortège des vices et des vertus "; il illustre l'ensemble par une pensée de Turgot : "La liberté, c'est le droit de faire tout ce qui n'est pas contraire au droit d'autrui ». Une formule dont on pourrait avoir, en 1943, plusieurs lectures. Dans le bandeau supérieur du carton, figure une balance sur fond de flammes sur laquelle G. Devêche ne fait aucun commentaire. Une référence au jugement dernier ?

\section{Le second projet}

C'est celui que Jean Chazal retiendra. On ne peut le dater avec exactitude, vraisemblablement 1944, mais avant ou après la libération de Paris ? 


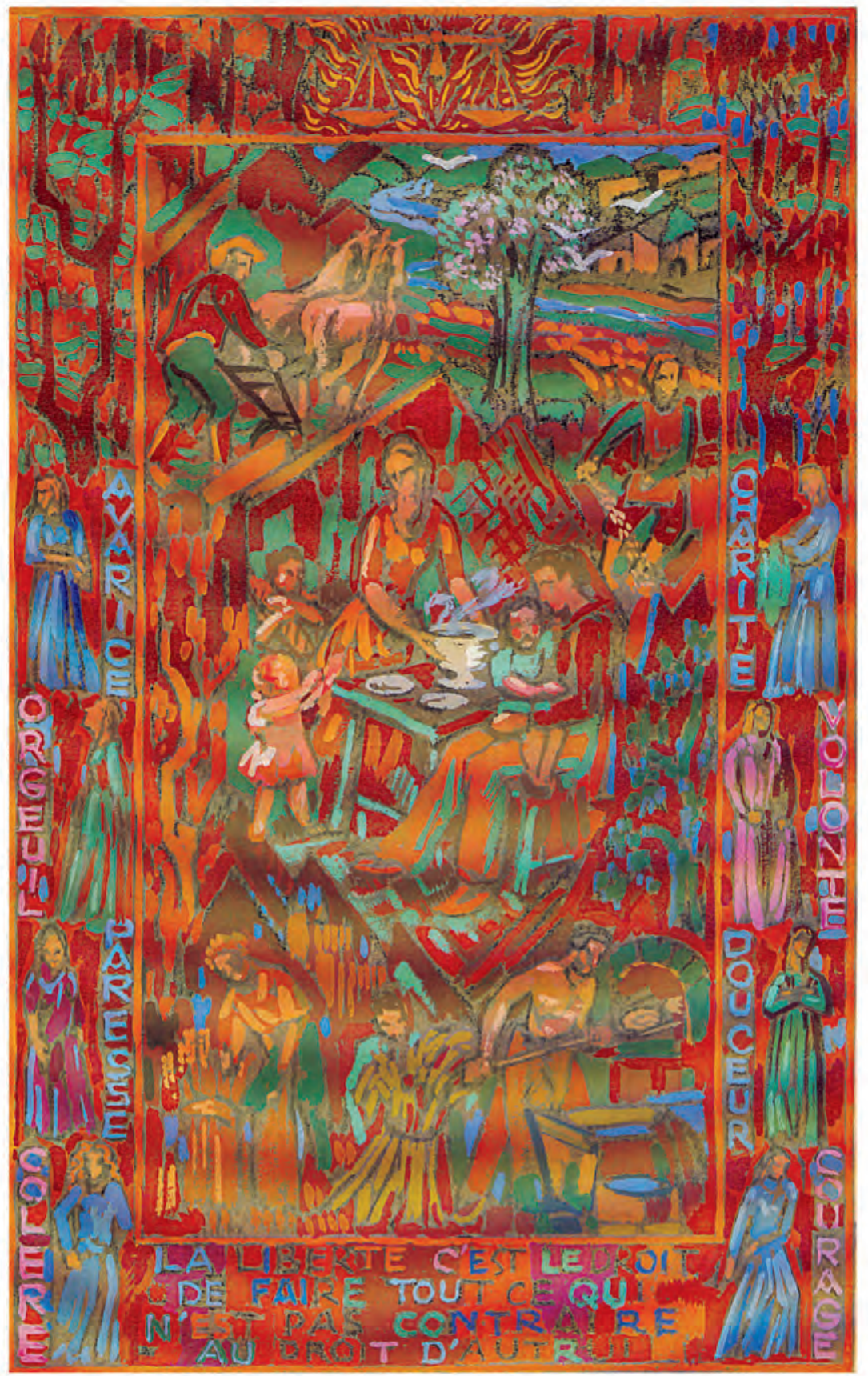

Georges Devêche, tapisserie pour le tribunal pour enfants de Paris.

Premier projet. Collection de l'auteur 


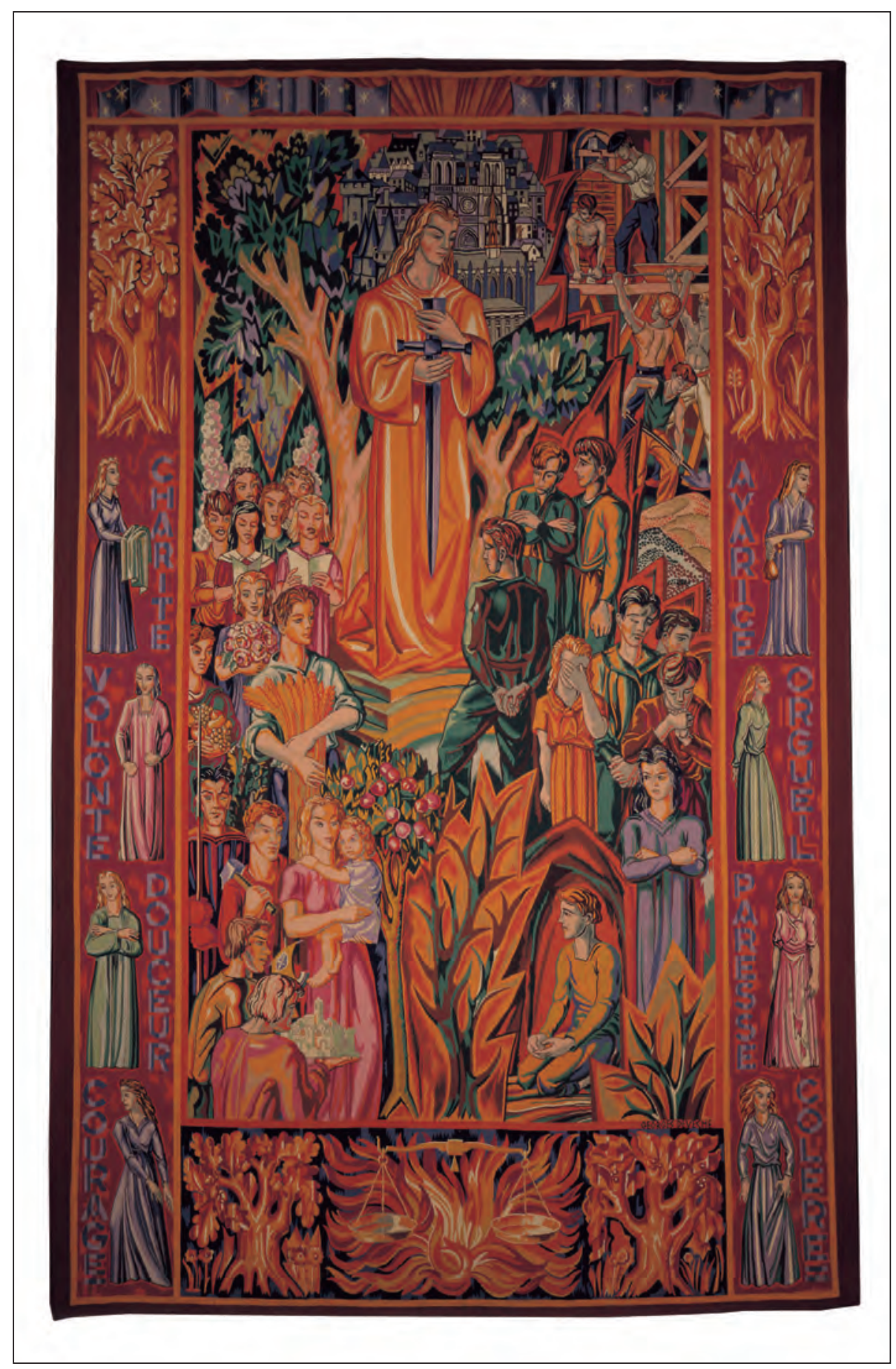

Georges Devêche, tapisserie pour le tribunal pour enfants de Paris.

Cliché Michel Basdevant 
Georges Devêche l'intitule La France défend la cité en distribuant la Justice ; la référence à la Patrie est évidente. La cité est représentée en haut du carton par la ville de Paris, où se détachent Notre-Dame et le Palais de Justice. L'œuvre s'organise autour d'un personnage central, debout sur une sorte de piédestal. Il porte de longs cheveux blonds, une longue tunique orangée sur laquelle se détache un glaive en forme de croix. Les bons sujets sont à droite, épanouis : une mère portant son enfant dans les bras, proche d'un arbre porteur de fruits, des gens au travail, des jeunes gens, filles et garçons, chantant au milieu des fleurs et des fruits ; une vision de paradis qui n'est pas sans évoquer Giotto. Au bas, G. Devêche fait figurer deux personnages qui apparaissent comme des donateurs, image courante dans la peinture votive médiévale ; l'un porte une caravelle, l'autre une cité, symbole vraisemblable de la ville de Paris, qui, rappelons-le, fut à l'origine de la commande de la tapisserie. Dans la partie gauche, deux personnages, des adolescents peut-être, dans une cellule ; leur attitude évoque la peine, le repentir, la soumission. Aux arbres remplis de fleurs de la partie droite se substituent des buissons d'épineux, des personnages aux visages indistincts que Georges Devêche appelle "les justiciables "; ils semblent écouter une sentence. En haut, à gauche, des échafaudages, des ouvriers au travail " construisent la cité ", suivant la formule de G. Devêche. Ils ne sont pas sans évoquer les bâtisseurs de cathédrales. Bien que les vices et les vertus aient disparu dans les bandeaux, ainsi que la balance et les flammes, la référence au jugement dernier se précise. La citation de Turgot a été supprimée et l'artiste intitule son second projet : « La France donne l'exemple à ceux qui ont failli à leur devoir ; la cité image de la civilisation ne peut vivre sans cette protection vigilante contre les mauvais sujets. »

\section{Le projet définitif, celui de 1946}

Les modifications par rapport au projet précédent sont mineures [ $c f$. illustration p. 151]. Il ne s'agit plus d'un carton, mais d'une œuvre tissée d'environ $18 \mathrm{~m} 2$. Les couleurs sont plus contrastées, les dessins plus lisibles, les traits plus définitifs. La référence au jugement dernier est très clairement renforcée. Les vices et les vertus apparaissent dans les bor- 
dures, mais elles sont inversées par rapport au premier projet : les vertus sont à droite, les vices à gauche. Nous sommes plus dans la tradition picturale des jugements derniers. La balance sur fond de flammes réapparait, mais cette fois elle figure en bas de la tapisserie, position plus conforme à la place traditionnelle de l'enfer. Le personnage central, que Georges Devêche, dans son projet précédent, identifie à la France, n'est pas sans évoquer le Christ avec le glaive que l'on trouve dans les jugements derniers de Memling, de Fra Angelico. Cette image peut se référer à l'apocalypse de Saint Jean ou à l'Évangile de Saint Mathieu, où le Christ apparaît au moment du jugement dernier ; c'est à lui que Dieu le père a remis le pouvoir de choisir les élus et les damnés.

On ne peut, derrière cette image de la justice, s'empêcher de voir celle du juge des enfants, dont la récente ordonnance du 2 février 1945 a fait le pilier, la clé de voûte de la réforme. À ses pieds, trois adolescents, dans une attitude d'attente, d'inquiétude attendent le prononcé de la décision. Un peu plus bas, un groupe de cinq jeunes, trois garçons et deux filles, nous fait face ; ils connaissent la décision : un garçon au visage ironique ? tourmenté ? un autre de profil, un troisième les mains jointes dans une attitude de prière, une fille les yeux cachés dans les mains masque son inquiétude, son désarroi, alors qu'une autre les bras croisés, le visage fermé, porte son regard sur une autre partie de la tapisserie, celle où la mère et l'enfant se trouvent parmi les justes. Encore plus bas, plus près des flammes, le cachot du précédent projet laisse la place à une sorte de grotte entourée de buissons épineux, où un adolescent isolé, à genoux, semble en pénitence. La partie droite de la tapisserie n'a pas changé. Ce sont les bons sujets, les justes, les élus. Ils apparaissent là comme l'exemple à suivre.

Cette œuvre élaborée dans une symbolique toute religieuse autour des notions de péché, de juste et de coupable, correspond-elle au discours qui fonde, pendant la guerre et au lendemain de la guerre, les orientations de la justice des mineurs?

Malgré une courbe de la délinquance juvénile en hausse manifeste depuis 1941, nous sommes, en 1945, dans un moment de libération nationale, et le regard que l'on porte sur le mineur délinquant est plein de 
(9) Jean Chazal,

Les enfants devant leurs juges, Éditions familiales françaises, Paris, 1946, p. 49 .

(10) Jean Chazal, "L'action humaine du juge des enfants”, Revue de l'Éducation surveillée, 1946, nº 3, p. 4.

(11) Jean Chazal, "L'enfant devant la sanction judiciaire", Sauvegarde de l'enfance, 1949, p. 19-20. mansuétude : c'est une victime de la guerre, il a été mal éduqué et l'ordonnance de 1945, nous l'avons vu, ne privilégiera ni la sanction, ni l'incarcération. On ne parlera plus de remoralisation comme en 1940, mais de rééducation, bien qu'entre les deux termes la différence ne soit pas manifeste. La sanction n'est pas à l'ordre du jour. La réforme est éducative ou ré-éducative à l'égard de ceux qui ont été mal élevés. N'oublions pas que tout ce projet a été élaboré et mis en forme pendant la période de la guerre. Jean Chazal a été l'interlocuteur privilégié de Georges Devêche. Leurs rencontres entre 1943 et 1946, selon André Devêche, n'ont pas donné lieu à correspondance, tous deux vivant à Paris. Jean Chazal a toutefois beaucoup écrit pendant cette période, et nous avons recherché dans ses nombreux articles ce qu'il disait de la justice des mineurs, du rôle du juge des enfants, quel regard il portait sur la délinquance juvénile.

C'est l'image de l'inadaptation sociale qui est dominante. "Pour les mineurs délinquants, écrit J. Chazal en 1946, il n'existe pas de différences psychologiques avec ceux qui ne le sont pas ; c'est un problème d'inadaptation à la vie sociale, familiale ; il importe d'obtenir leur adaptation en les éduquant, mais pour cela, il est souvent indispensable de les soigner. " ${ }^{(9)}$ Le rôle du juge des enfants, plus que de condamner, est d'orienter l'enfant dans la voie éducative. "Son action doit ouvrir la voie à celle de l'éducateur et du médecin. " ${ }^{(10)}$ Nous sommes pleinement dans la logique de l'ordonnance du 2 février 1945 qui, dans son exposé des motifs, évoque pour les mineurs la présomption d'irresponsabilité pénale. "Ce n'est pas, ajoute J. Chazal, le degré de responsabilité personnelle et intime du jeune délinquant qui importe, ce sont les causes mêmes de son comportement psychique, somatique, familial, social. [...] Si philosophiquement l'idée de responsabilité naît d'une liberté portant elle-même son propre commencement, le mineur délinquant n'est ni libre, ni responsable. " Dans ce même article, il réfute l'image du juge des enfants justicier : "Il ne s'agit pas d'expiation, d'exemplarité de la peine, celles-ci sont réservées aux adultes, l'ensemble de la mission des juges des enfants est surtout de pénétrer la personnalité du jeune délinquant. » ${ }^{(11)}$ 
Au propos moral teinté de religion qui semble être celui de G. Devêche dans sa tapisserie, se substitue un discours plus humaniste, plus scientifique, qui prend de la distance avec les notions du bien et du mal, du bon et du méchant. Si la peine doit être exceptionnelle pour le mineur, suivant l'ordonnance du 2 février 1945, le juge Chazal ne l'exclut pas pour ceux qu'il qualifie de pervers. Évoquant les mineurs criminels, il lui arrive de se référer à des images qui ne sont pas sans lien avec le jugement dernier : «Pesons les âmes des jeunes criminels, il en est parmi eux qu'une mesure éducative peut sauver. ${ }^{(12)}$

La tapisserie demeure dans une logique de punition. À l'égal des tympans de quelques cathédrales médiévales (Bourges, Autun...) qui représentent le jugement dernier, l'image doit avoir une fonction didactique et simpliste pour le pécheur : le ciel, l'enfer. Le jeune délinquant qui comparait face à ses juges se trouve face à la tapisserie ; elle est derrière le siège du tribunal. Le propos en est simple : il y a les bons, il y a les méchants, la justice punit les méchants.

N'était-il pas de la volonté de Georges Devêche, et peut-être de Jean Chazal, suite à la période de guerre où la frontière entre ce qui était permis et ce qui était défendu était devenue très floue, de préciser pour les mineurs la force et la place de la loi ? Enfin, ultime remarque, n'y avaitil pas chez les deux hommes, chrétiens convaincus, la volonté plus ou moins consciente de réintroduire le Christ dans les prétoires? 